\title{
Synthesis of $(E)$-1,4-diaryl-2-butene-1,4-diones
}

\author{
Chieh-Kai Chan and Meng-Yang Chang* \\ Department of Medicinal and Applied Chemistry, Kaohsiung Medical University, Kaohsiung 807, \\ Taiwan \\ E-mail: mychang@kmu.edu.tw
}

DOI: https://doi.org/10.24820/ark.5550190.p009.878

\begin{abstract}
We report a facile route for the preparation of symmetric and unsymmetric $(E)$-1,4-diaryl-2-butene1,4-diones 3 by a two-step route, including (1) nucleophilic substitution of $\mathbf{1}$ with sulfinic acid sodium salts, and (2) $\mathrm{K}_{2} \mathrm{CO}_{3}$ mediated alkylation of $\beta$-ketosulfones 4 with $\mathbf{1}$ followed by sequential desulfonylation of the resulting 1,4-diketones $\mathbf{5}$ in acetone. These products were obtained in high yields.
\end{abstract}

Keywords: Enedione, ketosulfone, quinoxaline, condensation

\section{Introduction}

$\beta$-Ketosulfones are key synthetic intermediates that can easily be prepared through direct nucleophilic substitution of $\alpha$-haloacetophenones, aerobic oxysulfonylation of functionalized alkenes and terminal alkynes and oxidation of $\alpha$-mercapto ketones in organic and medicinal fields. ${ }^{1-}$

6 Notably, a number of examples have been reported for versatile functional group transformations. ${ }^{7-29}$ The diversified skeletons include (1) acyclic diketones, ${ }^{7} \alpha$-methenyl ketones, ${ }^{8-}$ 9 and styrylsulfones, ${ }^{15}$ (2) monocyclic sulfones, ${ }^{10}$ cyclopropanes, ${ }^{16}$ triazoles, ${ }^{11}$ pyrazoles, ${ }^{12-13}$ dihydrofurans, ${ }^{14,19}$ tetrahydrofurans,${ }^{17}$ tetrahydropyrans, ${ }^{18}$ pyrroles,${ }^{20}$ furans, ${ }^{21-22}$ benzenes,${ }^{23}$ and pyridazines ${ }^{24}$ (3) bicyclic benzosuberans, ${ }^{25}$ tetralins, ${ }^{26}$ and naphthalenes, ${ }^{27}$ (4) tricyclic phenanthrenes, ${ }^{28}$ and (5) tetracyclic phenanthrofurans. ${ }^{29}$ 

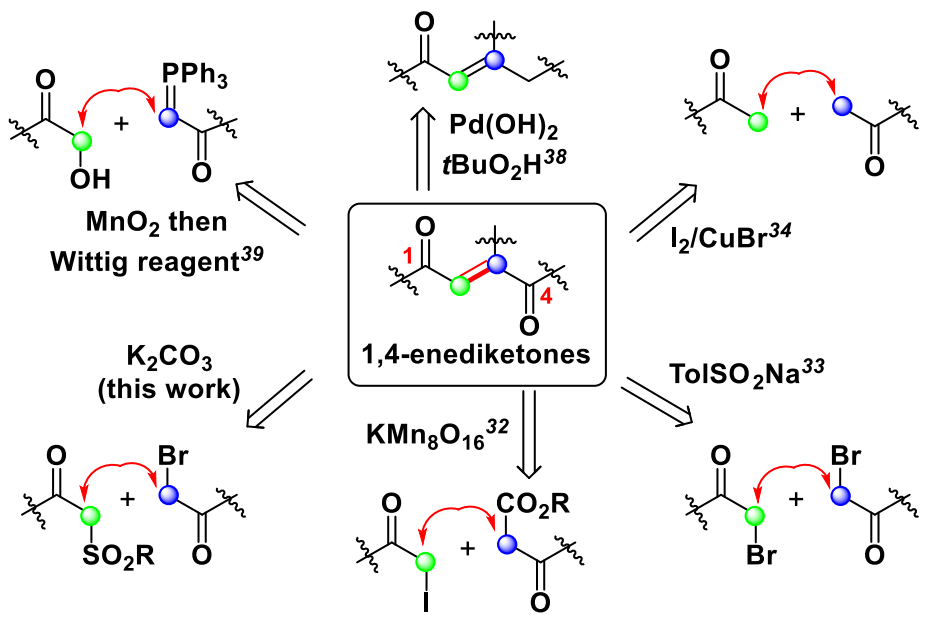

Scheme 1. Synthetic routes toward $(E)$-ene-1,4-diones

\section{Results and Discussion}

In continuation of the investigation on synthetic applications of $\beta$-ketosulfone, this study develops a facile synthesis of (E)-1,4-diaryl-2-butene-1,4-diones via $\mathrm{K}_{2} \mathrm{CO}_{3}$ mediated alkylative desulfonylation of $\beta$-ketosulfone with $\alpha$-haloacetophenone. As shown in Scheme 1, ene-1,4diketone is a versatile building block in the synthesis of bioactive molecules. ${ }^{30-31}$ A number of articles have highlighted the fascinating development of core 1,4 -enedione, ${ }^{32-39}$ including $\mathrm{KMn}_{8} \mathrm{O}_{16}$ catalyzed reaction of $\beta$-ketoesters and $\alpha$-iodoacetophenones, ${ }^{32}$ the direct nucleophilic substitution of $\alpha$-haloacetophenones and $\mathrm{TolSO}_{2} \mathrm{Na},{ }^{33}$ iodine/copper complex or hypervalent iodine mediated oxidative self- or cross-coupling of methyl ketones, ${ }^{35-37} \mathrm{Pd}(\mathrm{OH})_{2} / \mathrm{BuO}_{2} \mathrm{H}$ promoted allylic oxidation of enones, ${ }^{38}$ and the tandem reaction of $\alpha$-hydroxyacetophenones with $\mathrm{MnO}_{2}$ and Wittig reagent. ${ }^{39}$ Kong et al. recently reported a one-pot facile method for the synthesis of symmetric and unsymmetric ene-1,4-diones with $E$-form selectivity from the diversified $\alpha$-bromoacetophenones though a sodium sulfinate $(0.5 \mathrm{eq})$ mediated reaction in the presence of $\mathrm{K}_{2} \mathrm{CO}_{3}(1.5 \mathrm{eq})$ at $\mathrm{rt}$ for 12 $\mathrm{h}$, and in situ the generated $\beta$-ketosulfone played a key intermediate in the transformation (Scheme 2 and eq 1). ${ }^{33}$ 

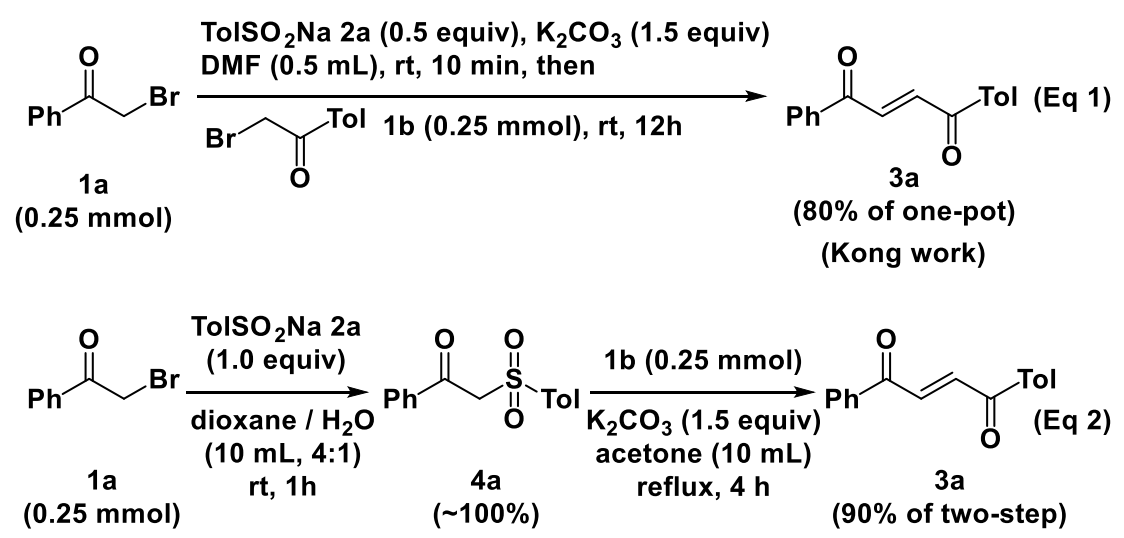

Scheme 2. Synthetic sequence of unsymmetrical ene-1,4-diones.

Although this one-pot route is mild, simple and convenient, the probability for two incomplete conversions should increase, especially for the formation of an unsymmetric skeleton of ene-1,4dione, including (1) a nucleophilic substitution of $\alpha$-bromoacetophenone (1a) with $\mathrm{TolSO}_{2} \mathrm{Na}(\mathbf{2 a}$, 0.5 equiv) in the presence of $\mathrm{K}_{2} \mathrm{CO}_{3}$ (1.5 equiv) affording $\beta$-ketosulfone and followed by (2) a desulfonylative elimination of the resulting $\beta$-ketosulfone with $\alpha$-bromo 4-methylacetophenone (1b, 1.0 equiv) producing ene-1,4-dione 3a. Under a highly concentrated reaction mixture (1.5 M, based on all substrates/DMF), we assumed that a different solubility of substrates should perform a competitive reaction easily during the overall process. However, this domino route often had other drawbacks, such as the use of a high boiling point solvent, and moderate isolated yields. Inspired by this route and with our interest in exploring practical applications of $\beta$-ketosulfones, herein we report the synthesis of symmetric and unsymmetric $(E)$-1,4-Diaryl-2-butene-1,4-diones by the conventional step-by-step route (Scheme 2 and eq 2). Initially, the nucleophilic substitution of $\mathbf{1 a}$ with 1.0 equivalent of $\mathbf{2 a}$ provided $\mathbf{4} \mathbf{a}$ in a quantitative yield after the recrystallization process.

Following a stepwise sequence, the $\mathrm{K}_{2} \mathrm{CO}_{3}$ (1.5 equiv) mediated reaction of $\mathbf{4 a}$ and $\mathbf{1 b}$ (1.0 equiv) produced 3a (46\%) and $\mathbf{5 a}(43 \%)$ with a yield ratio of $1: 1$ at $\mathrm{rt}$ for $4 \mathrm{~h}$, as shown in Table 1 and entry 1 . By an elongated time $(3 \rightarrow 20 \mathrm{~h})$, the yield of 3a (52\%) increased slightly and 33\% yield of $\mathbf{5 a}$ was formed (entry 2). To elevate the reaction temperature ( $\mathrm{rt} \rightarrow$ reflux), 3a was isolated in a $90 \%$ yield at $4 \mathrm{~h}$ (entry 3 ). Compared with the reaction temperature and time, the refluxing condition could enhance the yield of 3a. To combine the reaction condition of the reflux temperature $\left(56^{\circ} \mathrm{C}\right)$ and elongating time $(20 \mathrm{~h})$, the yield of $\mathbf{3 a}$ was decreased $(90 \% \rightarrow 72 \%$, entry 4). When the equivalent of $\mathrm{K}_{2} \mathrm{CO}_{3}$ was doubled, no obvious changes occurred (entry 5). Changing the solvent to THF, only the starting material $4 \mathbf{a}$ was recovered in an $84 \%$ yield due to the low solubility of $\mathrm{K}_{2} \mathrm{CO}_{3}$ in THF (entry 6). Controlling the reflux $\left(56{ }^{\circ} \mathrm{C}\right)$ and time $(4 \mathrm{~h})$ condition, other inorganic bases have been examined in entries 7-9. Changing $\mathrm{K}_{2} \mathrm{CO}_{3}$ to $\mathrm{Li}_{2} \mathrm{CO}_{3}, \mathrm{Na}_{2} \mathrm{CO}_{3}$, or $\mathrm{Cs}_{2} \mathrm{CO}_{3}$, these yields of $\mathbf{3 a}$ did not exhibit any obvious changes. Further variations of the reaction parameters such as the organic bases were carried out (entries 10-11). However, treatment of 4a with DBU and DABCO in THF at reflux for $4 \mathrm{~h}$ afforded $\mathbf{5 a}$ in low yields (10\% and 6\%). In entries 10-11, major 4a was recovered and no isolation of $\mathbf{3 a}$ was observed. On the basis of a higher yield, 
we believe that the combination of $\mathrm{K}_{2} \mathrm{CO}_{3} /$ refluxing acetone/4 $\mathrm{h}$ should be an optimal reaction condition for the formation of 3a. With the facile reaction condition in hand (Table 1, Entry 3), we further explored the conversion of other substrate scopes, and the results are shown in Table 2. $\mathrm{K}_{2} \mathrm{CO}_{3}$-mediated alkylative desulfonylation of $\beta$-ketosulfones $4 \mathbf{a}-\mathbf{f}\left(\mathrm{Ar}=4-\mathrm{MeOC}_{6} \mathrm{H}_{4}\right.$, Tol, 4$\mathrm{PhC}_{6} \mathrm{H}_{4}$; and $\mathrm{R}=$ Tol, $\left.\mathrm{Ph}, \mathrm{Me}\right)$ and $\alpha$-bromoacetophenones 1a-1i $\left(\mathrm{Ar}^{1}=\right.$ Tol, $\mathrm{Ph}, 3-\mathrm{MeOC}_{6} \mathrm{H}_{4}, 4-$ $\mathrm{FC}_{6} \mathrm{H}_{4}, 4-\mathrm{MeOC}_{6} \mathrm{H}_{4}, 4-\mathrm{PhC}_{6} \mathrm{H}_{4}, 2,4-(\mathrm{MeO})_{2} \mathrm{C}_{6} \mathrm{H}_{3}, 2,5-(\mathrm{MeO})_{2} \mathrm{C}_{6} \mathrm{H}_{3}$, 2-naphthyl) in acetone at reflux provided 3a-r in a yield range of $86 \%$ 94\%. The structures of $\mathbf{3 n}$ and $\mathbf{3 r}$ were determined by single-crystal X-ray crystallography. ${ }^{41}$

Table 1. Optimal conditions ${ }^{\mathrm{a}}$

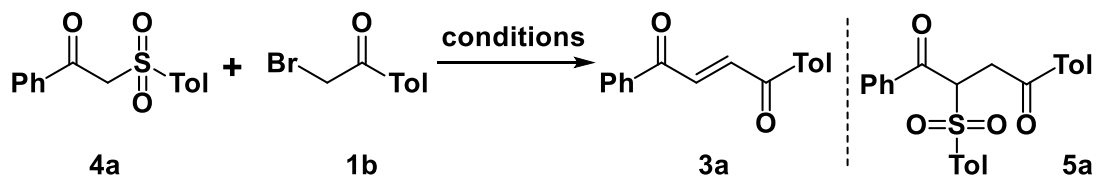

\begin{tabular}{cllccc}
\hline Entry & Base (equiv) & Solvent & Temp $\left({ }^{\circ} \mathrm{C}\right)$ & Time $(\mathrm{h})$ & $\mathbf{3 a}(\%)^{\mathrm{b}}$ \\
\hline 1 & $\mathrm{~K}_{2} \mathrm{CO}_{3}(1.5)$ & acetone & 25 & 4 & $46(43)^{\mathrm{c}}$ \\
2 & $\mathrm{~K}_{2} \mathrm{CO}_{3}(1.5)$ & acetone & 25 & 20 & $52(33)^{\mathrm{c}}$ \\
3 & $\mathrm{~K}_{2} \mathrm{CO}_{3}(1.5)$ & acetone & 56 & 4 & 90 \\
4 & $\mathrm{~K}_{2} \mathrm{CO}_{3}(1.5)$ & acetone & 56 & 20 & 72 \\
5 & $\mathrm{~K}_{2} \mathrm{CO}_{3}(3.0)$ & acetone & 56 & 4 & 85 \\
6 & $\mathrm{~K}_{2} \mathrm{CO}_{3}(1.5)$ & THF & 67 & 4 & $-{ }^{\mathrm{d}}$ \\
7 & $\mathrm{Li}_{2} \mathrm{CO}_{3}(1.5)$ & acetone & 56 & 4 & 80 \\
8 & $\mathrm{Na}_{2} \mathrm{CO}_{3}(1.5)$ & acetone & 56 & 4 & 86 \\
9 & $\mathrm{Cs}_{2} \mathrm{CO}_{3}(1.5)$ & acetone & 56 & 4 & 88 \\
10 & $\mathrm{DBU}(1.5)$ & THF & 67 & 4 & $-{ }^{\mathrm{d}}$ \\
11 & $\mathrm{DABCO}(1.5)$ & THF & 67 & 4 & $-{ }^{\mathrm{d}}$ \\
\hline
\end{tabular}

${ }^{\mathrm{a}}$ Reaction was run with $\mathbf{1 a}(0.25 \mathrm{mmol}), \mathbf{1 b}\left(1.0\right.$ equiv) and solvent $(10 \mathrm{~mL}){ }^{\mathrm{b}}$ Isolated yields. ${ }^{\mathrm{c}}$ Isolated yield of 5a. ${ }^{\mathrm{d}} \mathbf{3 a}$ was recovered (for entry 6, 84\%; for entry 10, 86\%; for entry 11, 86\%).

The yield of $\mathbf{3}$ did not change much as the function of the structures of $\mathbf{4}$ and $\mathbf{1}$ under these conditions. Changing the base to 1.0 equivalent of LDA (from 1.5 equivalent of $\mathrm{K}_{2} \mathrm{CO}_{3}$ ) and decreasing the temperature to $-78^{\circ} \mathrm{C}$ (from reflux), the alkylation of $\mathbf{4}(\mathrm{Ar}=; \mathbf{4 a}, \mathrm{Ph} ; \mathbf{4 c}, \mathrm{Tol} ; \mathbf{4 b}, 4-$ $\left.\mathrm{MeOC}_{6} \mathrm{H}_{4} ; \mathbf{4 g}, 2,4-(\mathrm{MeO})_{2} \mathrm{C}_{6} \mathrm{H}_{3}\right)$ with $1\left(\mathrm{Ar}^{1}=; \mathbf{1 b}\right.$, Tol; 1g, 3,4-(MeO) $\left.{ }_{2} \mathrm{C}_{6} \mathrm{H}_{3} ; \mathbf{1 e}, 4-\mathrm{MeOC}_{6} \mathrm{H}_{4}\right)$ provided 5a-e in a yield range of $73 \sim 81 \%$ at $-78{ }^{\circ} \mathrm{C}$ for $4 \mathrm{~h}$, as shown in Table 3. Compared with the $\mathrm{K}_{2} \mathrm{CO}_{3}$ /acetone/reflux condition, we found that the LDA/THF/-78 ${ }^{\circ} \mathrm{C}$ system could perform only the generation of sulfonyl 1,4-diketones $\mathbf{5}$ and no isolation of ene-1,4-diones $\mathbf{3}$. 
Table 2. Synthesis of 3a-ra

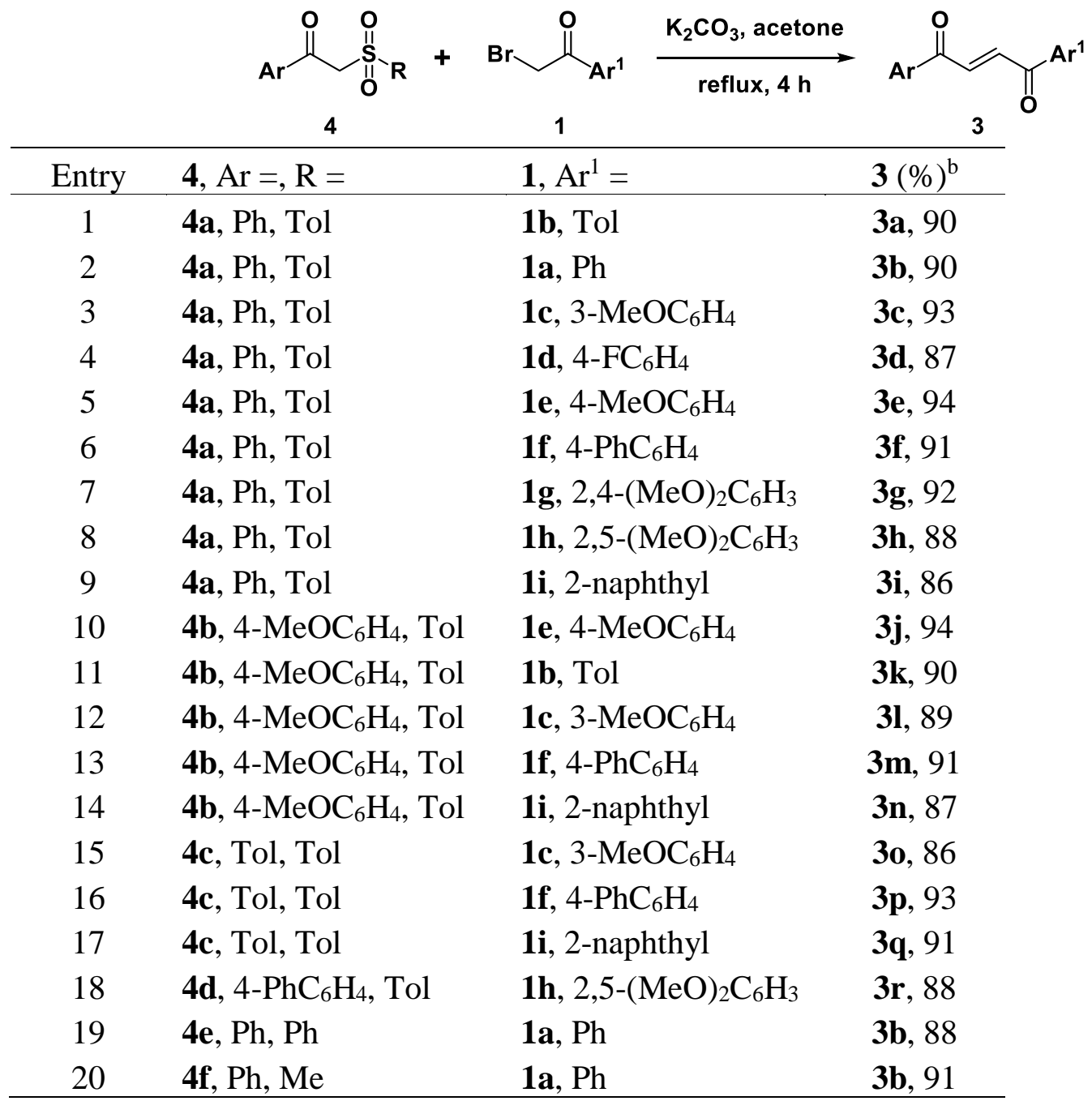

The synthesis of $\mathbf{3}$ was run with $4(0.25 \mathrm{mmol}), \mathbf{1}(0.25 \mathrm{mmol})$, acetone $(10 \mathrm{~mL}), 4 \mathrm{~h}$, reflux. bisolated yields.

As an extension of this method, we were able to execute a synthesis of quinoxaline, as shown in Scheme 3. Quinoxaline was a versatile scaffold for useful synthetic intermediates. ${ }^{41-42}$ It was also known to exhibit versatile biological activities. ${ }^{43-44}$ On the basis of these significant characteristics, many protocols have been developed for the synthesis of quinoxalines. According to reported literature, the most popular procedures are derived from the condensation of 1,2diaminobenzenes with a number of polar ortho-carbon units, such as aldehydes, ketones, 1,2diketones, epoxides, vicinal diols, diazoketone, alkenes, and alkyne. ${ }^{45}$ Among these starting substrates, only one example on the skeleton of 1,4-enediketone has been reported for the formation of quinoxaline. ${ }^{46}$ Furthermore, condensation of $\mathbf{3 b}$ with 1,2-diaminobenzenes $\mathbf{6 a - d}$ in dioxane for $4 \mathrm{~h}$ at reflux provided quinoxalines $\mathbf{7 a - d}(78 \%-86 \%)$ and acetophenone $(\mathbf{8})$ via a tandem process 
of the condensation of $\mathbf{3 b}$ and $\mathbf{6}$, the intramolecular Michael addition of intermediate $\mathbf{I}$, and the retro-aldol reaction of intermediate II.

Table 3. Alkylation of $\mathbf{4}$ with $\mathbf{1}^{\mathrm{a}}$<smiles>O=C([AlH2])CS(=O)(=O)[O-]</smiles>

4

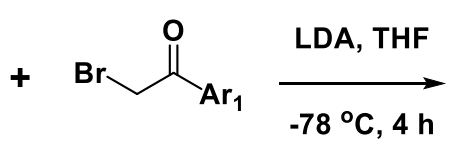

1

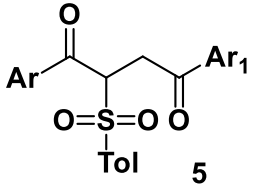

\begin{tabular}{clll}
\hline Entry & $\mathbf{4}, \mathrm{Ar}=$ & $\mathbf{1}, \mathrm{Ar}^{1}=$ & $\mathbf{5}(\%)^{\mathrm{b}}$ \\
\hline 1 & $\mathbf{4 a}, \mathrm{Ph}$ & $\mathbf{1 b}, \mathrm{Tol}$ & $\mathbf{5 a}, 81$ \\
2 & $\mathbf{4 c}, \mathrm{Tol}$ & $\mathbf{1 b}, \mathrm{Tol}$ & $\mathbf{5 b}, 75$ \\
3 & $\mathbf{4 c}, \mathrm{Tol}$ & $\mathbf{1 g}, 2,4-(\mathrm{MeO})_{2} \mathrm{C}_{6} \mathrm{H}_{3}$ & $\mathbf{5 c}, 77$ \\
4 & $\mathbf{4 b}, 4-\mathrm{MeOC} \mathrm{H}_{4}$ & $\mathbf{1 g}, 2,4-(\mathrm{MeO})_{2} \mathrm{C}_{6} \mathrm{H}_{3}$ & $\mathbf{5 d}, 73$ \\
5 & $\mathbf{4 g}, 2,4-(\mathrm{MeO})_{2} \mathrm{C}_{6} \mathrm{H}_{3}$ & $\mathbf{1 e}, 4-\mathrm{MeOC}_{6} \mathrm{H}_{4}$ & $\mathbf{5 e}, 76$ \\
\hline
\end{tabular}

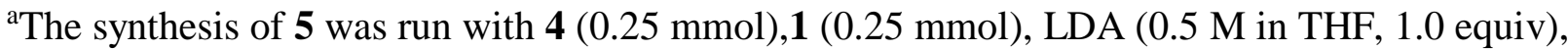
THF (5 mL). ' Isolated yields.
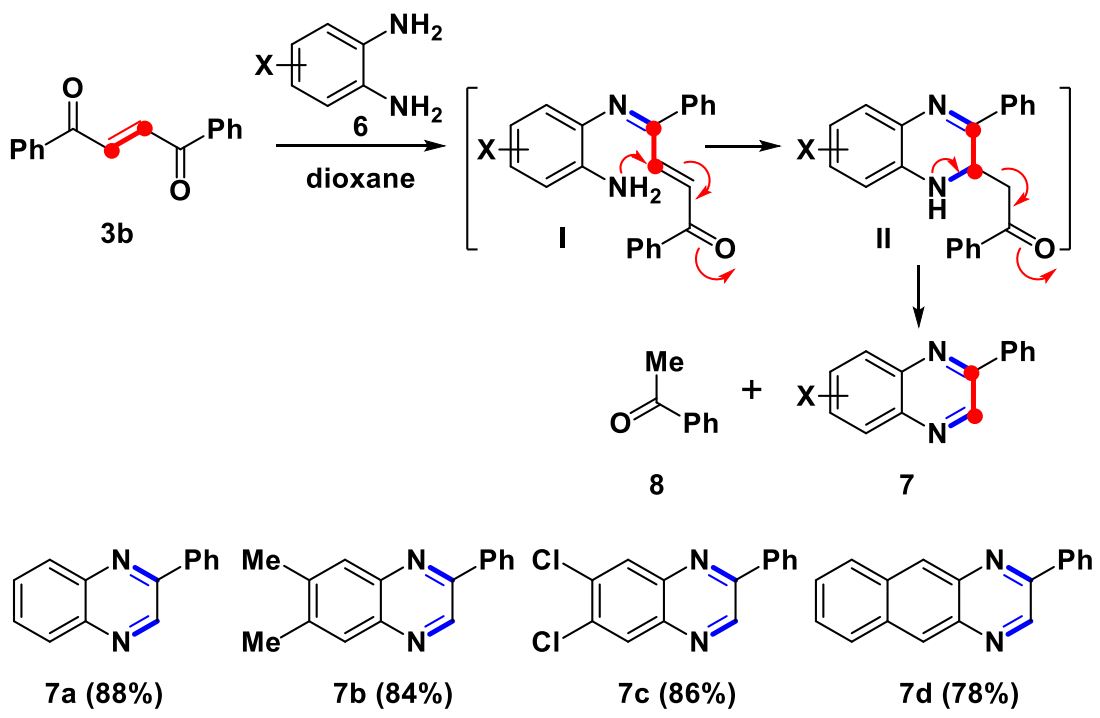

Scheme 3. Condensation of $\mathbf{3 b}$ with 1,2-diaminobenzenes 6a-d.

\section{Conclusions}

We have developed a mild and facile synthesis of substituted symmetric and unsymmetric ene-1,4diketones 3 in good yields by a two-step route, including (1) nucleophilic substitution of $\alpha$ bromoacetophenones $\mathbf{1}$ with sulfinic acid sodium salts $\mathbf{2}$ in a co-solvent of dioxane and water at $\mathrm{rt}$ 
for $1 \mathrm{~h}$, and (2) $\mathrm{K}_{2} \mathrm{CO}_{3}$ mediated alkylation of $\beta$-ketosulfones 4 with substituted $\alpha$ bromoacetophenones 1 followed by sequential desulfonylation of the resulting $\alpha$-sulfonyl 1,4diketones 5 in acetone at reflux for $4 \mathrm{~h}$. Morever, quinoxalines 7 have been synthesized from a condensation of ene-1,4-diketone $\mathbf{3 b}$ with 1,2-diaminobenzenes $\mathbf{6}$. Further investigation regarding the synthetic applications of $\beta$-ketosulfones will be conducted and published in due course.

\section{Experimental Section}

General. All other reagents and solvents were obtained from commercial sources and used without further purification. Reactions were routinely carried out under an atmosphere of dry nitrogen with magnetic stirring. Products in organic solvents were dried with anhydrous $\mathrm{MgSO}_{4}$ before concentration in vacuo. Melting points were determined with a SMP3 melting apparatus. ${ }^{1} \mathrm{H}$ and ${ }^{13}$ C NMR spectra were recorded on a Varian INOVA-400 spectrometer operating at 200/400 and at $100 \mathrm{MHz}$, respectively. Chemical shifts $(\delta)$ are reported in parts per million (ppm) and the coupling constants $(J)$ are given in Hertz. High resolution mass spectra (HRMS) were measured with a mass spectrometer Finnigan/Thermo Quest MAT 95XL. X-ray crystal structures were obtained with an Enraf-Nonius FR-590 diffractometer (CAD4, Kappa CCD). Elemental analyses were carried out with Heraeus Vario III-NCSH, Heraeus CHN-OS-Rapid Analyzer or Elementar Vario EL III.

A representative procedure for compounds 3a-r is as follows. A solution of sodium arenesulfinic acid salts $2(0.25 \mathrm{mmol})$ in $\mathrm{H}_{2} \mathrm{O}(2 \mathrm{~mL})$ was added to a solution of substituted $\alpha$ bromoacetophenones $1(0.25 \mathrm{mmol})$ in dioxane $(8 \mathrm{~mL})$ at $\mathrm{rt}$. The reaction mixture was stirred at $\mathrm{rt}$ for $1 \mathrm{~h}$ and the solvent was concentrated. The residue was diluted with water $(10 \mathrm{~mL})$ and the mixture was extracted with $\mathrm{CH}_{2} \mathrm{Cl}_{2}(3 \times 20 \mathrm{~mL})$. The combined organic layers were washed with brine, dried, filtered and evaporated to afford crude product $\mathbf{4 a - f}$ in nearly quantitative yields. Without further purification, $\mathrm{K}_{2} \mathrm{CO}_{3}(52 \mathrm{mg}, 0.377 \mathrm{mmol})$ was added to a solution of the resulting $4(\sim 0.25 \mathrm{mmol})$ in acetone $(8 \mathrm{~mL})$ at $\mathrm{rt}$. The reaction mixture was stirred at $\mathrm{rt}$ for $5 \mathrm{~min}$. $\alpha$ Bromoacetophenones 1a-i $(0.25 \mathrm{mmol})$ in acetone $(2 \mathrm{~mL})$ was added to the resulting reaction mixture at $\mathrm{rt}$. The reaction mixture was refluxed for $4 \mathrm{~h}$, cooled to $\mathrm{rt}$, and the solvent was concentrated. The residue was diluted with water $(10 \mathrm{~mL})$ and the mixture was extracted with $\mathrm{CH}_{2} \mathrm{Cl}_{2}$ (3 x $20 \mathrm{~mL}$ ). The combined organic layers were washed with brine, dried, filtered and evaporated to afford crude product. Purification on silica gel (hexanes/EtOAc 10/1 3/1) afforded compounds 3a-r.

1-Phenyl-4-p-tolylbut-2-ene-1,4-dione (3a). $\mathrm{R}_{f} 0.3$ (hexanes : EtOAc 6:1); Yield 90\% (56 mg); Colorless solid; mp 83-84 ${ }^{\circ} \mathrm{C}$ (recrystallized from hexanes and EtOAc); HRMS (ESI, $\mathrm{M}^{+}+1$ ) calcd for $\mathrm{C}_{17} \mathrm{H}_{15} \mathrm{O}_{2} 251.1072$, found 251.1075; ${ }^{1} \mathrm{H}$ NMR (400 MHz, $\left.\mathrm{CDCl}_{3}\right): \delta 8.08-8.04(\mathrm{~m}, 2 \mathrm{H}), 8.00$ $(\mathrm{s}, 2 \mathrm{H}), 7.97(\mathrm{~d}, J 8.0 \mathrm{~Hz}, 2 \mathrm{H}), 7.65-7.61(\mathrm{~m}, 1 \mathrm{H}), 7.55-7.50(\mathrm{~m}, 2 \mathrm{H}), 7.32(\mathrm{~d}, J 8.0 \mathrm{~Hz}, 2 \mathrm{H}), 2.44$ 
(s, 3H); ${ }^{13} \mathrm{C}$ NMR $\left(100 \mathrm{MHz}, \mathrm{CDCl}_{3}\right): \delta 189.87,189.25,144.93,136.92,135.26,134.73,134.41$, $133.77,129.58(2 \mathrm{x}), 129.12(2 \mathrm{x}), 128.84(4 \mathrm{x}), 21.74$.

1,4-Diphenylbut-2-ene-1,4-dione (3b). In Table 2, for entry 2, $\mathrm{R}_{f} 0.3$ (hexanes : EtOAc 6:1); Yield 90\% (53 mg); For entry 19, Yield 88\% (52 mg); For entry 20, Yield 91\% (54 mg); Colorless solid; mp 108-109 ${ }^{\circ} \mathrm{C}$ (recrystallized from hexanes and EtOAc); HRMS (ESI, $\mathrm{M}^{+}+1$ ) calcd for $\mathrm{C}_{16} \mathrm{H}_{13} \mathrm{O}_{2}$ 237.0916, found 237.0912; ${ }^{1} \mathrm{H}$ NMR (400 MHz, $\left.\mathrm{CDCl}_{3}\right): \delta 8.08-8.05(\mathrm{~m}, 4 \mathrm{H}), 8.02(\mathrm{~s}, 2 \mathrm{H}), 7.66-$ $7.62(\mathrm{~m}, 2 \mathrm{H}), 7.55-7.51(\mathrm{~m}, 4 \mathrm{H}) ;{ }^{13} \mathrm{C} \mathrm{NMR}\left(100 \mathrm{MHz}, \mathrm{CDCl}_{3}\right): \delta 189.81(2 \mathrm{x}), 136.85(2 \mathrm{x}), 135.09$ $(2 \mathrm{x}), 133.86(2 \mathrm{x}), 128.89(4 \mathrm{x}), 128.87(4 \mathrm{x})$.

1-(3-Methoxyphenyl)-4-phenylbut-2-ene-1,4-dione (3c). $\mathrm{R}_{f} 0.3$ (hexanes : EtOAc 4:1); Yield 93\% (62 mg); Colorless solid; mp 65-66 ${ }^{\circ} \mathrm{C}$ (recrystallized from hexanes and EtOAc); HRMS (ESI, $\mathrm{M}^{+}+1$ ) calcd for $\mathrm{C}_{17} \mathrm{H}_{15} \mathrm{O}_{3} 267.1021$, found 267.1020; ${ }^{1} \mathrm{H} \mathrm{NMR}\left(400 \mathrm{MHz}, \mathrm{CDCl}_{3}\right): \delta 8.07-8.03$ $(\mathrm{m}, 2 \mathrm{H}), 8.00(\mathrm{~s}, 1 \mathrm{H}), 7.99(\mathrm{~s}, 1 \mathrm{H}), 7.65-7.61(\mathrm{~m}, 2 \mathrm{H}), 7.57-7.50(\mathrm{~m}, 3 \mathrm{H}), 7.43(\mathrm{t}, J 8.0 \mathrm{~Hz}, 1 \mathrm{H})$, 7.19-7.16 (m, 1H), $3.88(\mathrm{~s}, 3 \mathrm{H}) ;{ }^{13} \mathrm{C} \mathrm{NMR}\left(100 \mathrm{MHz}, \mathrm{CDCl}_{3}\right): \delta 189.77,189.54,160.02,138.17$, 136.82, 135.10, 135.04, 133.83, 129.84, 128.86 (2x), 128.84 (2x), 121.62, 120.63, 112.65, 55.47.

1-(4-Fluorophenyl)-4-phenylbut-2-ene-1,4-dione (3d). $\mathrm{R}_{f} 0.3$ (hexanes : EtOAc 8:1); Yield 87\% (55 mg); Colorless solid; mp 102-103 ${ }^{\circ} \mathrm{C}$ (recrystallized from hexanes and EtOAc); HRMS (ESI, $\left.\mathrm{M}^{+}+1\right)$ calcd for $\mathrm{C}_{16} \mathrm{H}_{12} \mathrm{FO}_{2} 255.0821$, found $255.0817 ;{ }^{1} \mathrm{H} \mathrm{NMR}\left(400 \mathrm{MHz}, \mathrm{CDCl}_{3}\right): \delta 8.13-8.05$ (m, 4H), $8.01(\mathrm{~s}, 1 \mathrm{H}), 8.00(\mathrm{~s}, 1 \mathrm{H}), 7.66-7.62(\mathrm{~m}, 1 \mathrm{H}), 7.56-7.51(\mathrm{~m}, 2 \mathrm{H}), 7.23-7.17(\mathrm{~m}, 2 \mathrm{H}) ;{ }^{13} \mathrm{C}$ NMR $\left(100 \mathrm{MHz}, \mathrm{CDCl}_{3}\right): \delta 189.64,188.13,166.19$ (d, J $\left.255.5 \mathrm{~Hz}\right), 136.79,135.26,134.66$, 133.92, 133.31 (d, $J 3.1 \mathrm{~Hz}), 131.65,131.56,128.91$ (2x), 128.87 (2x), 116.13 (d, $J 22.0 \mathrm{~Hz}, 2 \mathrm{x}$ ). 1-(4-Methoxyphenyl)-4-phenylbut-2-ene-1,4-dione (3e). $\mathrm{R}_{f} 0.3$ (hexanes : EtOAc 4:1); Yield 94\% (63 mg); Colorless solid; mp 80-81 ${ }^{\circ} \mathrm{C}$ (recrystallized from hexanes and EtOAc); HRMS (ESI, $\mathrm{M}^{+}+1$ ) calcd for $\mathrm{C}_{17} \mathrm{H}_{15} \mathrm{O}_{3}$ 267.1021, found 267.1018; ${ }^{1} \mathrm{H} \mathrm{NMR}\left(400 \mathrm{MHz}, \mathrm{CDCl}_{3}\right.$ ): $\delta$ 8.09-8.01 $(\mathrm{m}, 4 \mathrm{H}), 8.02(\mathrm{~s}, 1 \mathrm{H}), 8.01(\mathrm{~s}, 1 \mathrm{H}), 7.65-7.61(\mathrm{~m}, 1 \mathrm{H}), 7.55-7.51(\mathrm{~m}, 2 \mathrm{H}), 7.01-6.98(\mathrm{~m}, 2 \mathrm{H}), 3.90$ $(\mathrm{s}, 3 \mathrm{H}) ;{ }^{13} \mathrm{C}$ NMR $\left(100 \mathrm{MHz}, \mathrm{CDCl}_{3}\right): \delta 189.95,187.94,164.22,136.94,135.29,134.39,133.78$, $131.34(2 \mathrm{x}), 129.97,128.86(4 \mathrm{x}), 114.12(2 \mathrm{x}), 55.56$.

1-Biphenyl-4-yl-4-phenylbut-2-ene-1,4-dione (3f). $\mathrm{R}_{f} 0.3$ (hexanes : EtOAc 6:1); Yield 91\% (71 $\mathrm{mg}$ ); Colorless solid; mp $155-156{ }^{\circ} \mathrm{C}$ (recrystallized from hexanes and EtOAc); HRMS (ESI, $\mathrm{M}^{+}+1$ ) calcd for $\mathrm{C}_{22} \mathrm{H}_{17} \mathrm{O}_{2} 313.1229$, found 313.1230; ${ }^{1} \mathrm{H} \mathrm{NMR}\left(400 \mathrm{MHz}, \mathrm{CDCl}_{3}\right): \delta 8.16-8.13$ $(\mathrm{m}, 2 \mathrm{H}), 8.09-8.07(\mathrm{~m}, 2 \mathrm{H}), 8.06(\mathrm{~s}, 1 \mathrm{H}), 8.05(\mathrm{~s}, 1 \mathrm{H}), 7.76-7.73(\mathrm{~m}, 2 \mathrm{H}), 7.66-7.62(\mathrm{~m}, 3 \mathrm{H}), 7.55-$ $7.40(\mathrm{~m}, 5 \mathrm{H}) ;{ }^{13} \mathrm{C}$ NMR $\left(100 \mathrm{MHz}, \mathrm{CDCl}_{3}\right): \delta 189.75,189.15,146.49,139.52,136.84,135.03$, 135.03, 134.92, 133.81, 129.47 (2x), 128.85 (2x), $128.85(4 x), 128.42,127.45(2 x), 127.25(2 x)$.

1-(2,4-Dimethoxyphenyl)-4-phenylbut-2-ene-1,4-dione (3g). $\mathrm{R}_{f} 0.2$ (hexanes : EtOAc 4:1); Yield 92\% (68 mg); Colorless solid; mp 105-106 ${ }^{\circ} \mathrm{C}$ (recrystallized from hexanes and EtOAc); HRMS (ESI, $\mathrm{M}^{+}+1$ ) calcd for $\mathrm{C}_{18} \mathrm{H}_{17} \mathrm{O}_{4} 297.1127$, found $297.1130 ;{ }^{1} \mathrm{H}$ NMR (400 $\mathrm{MHz}, \mathrm{CDCl}_{3}$ ): $\delta$ 8.03-8.01 (m, 2H), $7.93(\mathrm{dd}, J 0.8,15.2 \mathrm{~Hz}, 1 \mathrm{H}), 7.81$ (dd, $J 0.8,8.8 \mathrm{~Hz}, 1 \mathrm{H}), 7.79$ (dd, $J$ 0.8, $15.2 \mathrm{~Hz}, 1 \mathrm{H}), 7.60-7.56(\mathrm{~m}, 1 \mathrm{H}), 7.50-7.46$ (m, 2H), 6.54 (ddd, $J$ 0.8, 2.0, $8.8 \mathrm{~Hz}, 1 \mathrm{H}), 6.45$ (d, $J$ $2.0 \mathrm{~Hz}, 1 \mathrm{H}), 3.87(\mathrm{~s}, 3 \mathrm{H}), 3.84(\mathrm{~s}, 3 \mathrm{H}) ;{ }^{13} \mathrm{C} \mathrm{NMR}\left(100 \mathrm{MHz}, \mathrm{CDCl}_{3}\right): \delta 190.69,189.15,165.22$, 161.22, 140.52, 137.10, 133.37, 133.22, 131.99, 128.73 (2x), 128.62 (2x), 120.77, 105.68, 98.24, $55.62,55.49$. 
1-(2,5-Dimethoxyphenyl)-4-phenylbut-2-ene-1,4-dione (3h). $\mathbf{R}_{f} 0.2$ (hexanes : EtOAc 4:1); Yield 88\% (65 mg); Colorless viscous oil; HRMS (ESI, $\mathrm{M}^{+}+1$ ) calcd for $\mathrm{C}_{18} \mathrm{H}_{17} \mathrm{O}_{4} 297.1127$, found 297.1130; ${ }^{1} \mathrm{H} \mathrm{NMR}\left(400 \mathrm{MHz}, \mathrm{CDCl}_{3}\right): \delta 8.06-8.04(\mathrm{~m}, 2 \mathrm{H}), 7.89(\mathrm{~d}, J 15.2 \mathrm{~Hz}, 1 \mathrm{H}), 7.82(\mathrm{~d}, J$ 15.2 Hz, 1H), 7.65-7.60 (m, 1H), 7.54-7.49 (m, 2H), 7.28 (d, J 3.6 Hz, 1H), 7.10 (dd, J 3.6, 9.2 Hz, $1 \mathrm{H}), 6.95(\mathrm{~d}, J 9.2 \mathrm{~Hz}, 1 \mathrm{H}), 3.89(\mathrm{~s}, 3 \mathrm{H}), 3.82(\mathrm{~s}, 3 \mathrm{H}) ;{ }^{13} \mathrm{C} \mathrm{NMR}\left(100 \mathrm{MHz}, \mathrm{CDCl}_{3}\right): \delta 191.24$, 190.71, 153.71, 153.65, 139.87, 137.11, 133.60, 132.72, 128.89 (2x), 128.78 (2x), 127.95, 121.30, $114.19,113.30,56.28,55.87$.

1-Naphthalen-2-yl-4-phenylbut-2-ene-1,4-dione (3i). $\mathrm{R}_{f} 0.3$ (hexanes : EtOAc 6:1); Yield 86\% (61 mg); Colorless solid; mp 115-116 ${ }^{\circ} \mathrm{C}$ (recrystallized from hexanes and EtOAc); HRMS (ESI, $\mathrm{M}^{+}+1$ ) calcd for $\mathrm{C}_{20} \mathrm{H}_{15} \mathrm{O}_{2} 287.1072$, found 287.1075; ${ }^{1} \mathrm{H}$ NMR (400 MHz, $\left.\mathrm{CDCl}_{3}\right): \delta 8.60(\mathrm{~s}, 1 \mathrm{H})$, $8.21(\mathrm{~d}, J 15.2 \mathrm{~Hz}, 1 \mathrm{H}), 8.14-8.07(\mathrm{~m}, 4 \mathrm{H}), 8.01(\mathrm{~d}, J 8.4 \mathrm{~Hz}, 1 \mathrm{H}), 7.95(\mathrm{~d}, J 8.4 \mathrm{~Hz}, 1 \mathrm{H}), 7.90(\mathrm{~d}$, $J$ 8.0 Hz, 1H), 7.67-7.53 (m, 5H); $\left.{ }^{13} \mathrm{C} \mathrm{NMR} \mathrm{(100} \mathrm{MHz,} \mathrm{CDCl}_{3}\right): \delta 189.86,189.49,136.92,135.89$, $135.13,134.93$ (2x), 134.28, 133.88, 132.46, 131.16, 129.77, 129.05, 128.94 (2x), 128.90 (2x), $127.86,127.06,124.05$.

1,4-Bis-(4-methoxyphenyl)but-2-ene-1,4-dione (3j). $\mathrm{R}_{f} 0.2$ (hexanes : EtOAc 4:1); Yield 94\% (70 mg); Colorless solid; mp 161-162 ${ }^{\circ} \mathrm{C}$ (recrystallized from hexanes and EtOAc); HRMS (ESI, $\mathrm{M}^{+}+1$ ) calcd for $\mathrm{C}_{18} \mathrm{H}_{17} \mathrm{O}_{4} 297.1127$, found 297.1130; ${ }^{1} \mathrm{H}$ NMR (400 MHz, $\left.\mathrm{CDCl}_{3}\right): \delta 8.06(\mathrm{~d}, J$ $8.8 \mathrm{~Hz}, 4 \mathrm{H}), 8.01(\mathrm{~s}, 2 \mathrm{H}), 6.98(\mathrm{~d}, J 8.8 \mathrm{~Hz}, 4 \mathrm{H}), 3.89(\mathrm{~s}, 6 \mathrm{H}) ;{ }^{13} \mathrm{C} \mathrm{NMR}\left(100 \mathrm{MHz}, \mathrm{CDCl}_{3}\right): \delta$ $188.07(2 \mathrm{x}), 164.14(2 \mathrm{x}), 134.56(2 \mathrm{x}), 131.31(4 \mathrm{x}), 130.06(2 \mathrm{x}), 114.08(4 \mathrm{x}), 55.53(2 \mathrm{x})$.

1-(4-Methoxyphenyl)-4-p-tolylbut-2-ene-1,4-dione (3k). $\mathrm{R}_{f} 0.3$ (hexanes : EtOAc 4:1); Yield 90\% (63 mg); Colorless solid; mp 105-106 ${ }^{\circ} \mathrm{C}$ (recrystallized from hexanes and EtOAc); HRMS (ESI, $\mathrm{M}^{+}+1$ ) calcd for $\mathrm{C}_{18} \mathrm{H}_{17} \mathrm{O}_{3} 281.1178$, found $281.1175 ;{ }^{1} \mathrm{H}$ NMR $\left(400 \mathrm{MHz}, \mathrm{CDCl}_{3}\right.$ ): $\delta 8.07$ $(\mathrm{d}, J 8.8 \mathrm{~Hz}, 2 \mathrm{H}), 8.00(\mathrm{~s}, 2 \mathrm{H}), 7.97(\mathrm{~d}, J 8.0 \mathrm{~Hz}, 2 \mathrm{H}), 7.31(\mathrm{~d}, J 8.4 \mathrm{~Hz}, 2 \mathrm{H}), 6.98(\mathrm{~d}, J 8.8 \mathrm{~Hz}$, 2H), 3.89 (s, 3H), 2.44 (s, 3H); $\left.{ }^{13} \mathrm{C} \mathrm{NMR} \mathrm{(100} \mathrm{MHz,} \mathrm{CDCl}_{3}\right)$ : $\delta$ 189.40, 188.02, 164.17, 144.84, 134.91, 134.56, 131.31 (2x), 130.02, 129.55 (2x), 129.00 (2x), 127.81, 114.09 (2x), 55.54, 21.74. 1-(3-Methoxyphenyl)-4-(4-methoxyphenyl)but-2-ene-1,4-dione (3I). $\mathrm{R}_{f} 0.3$ (hexanes : EtOAc 4:1); Yield 89\% (66 mg); Colorless solid; mp 93-94 ${ }^{\circ} \mathrm{C}$ (recrystallized from hexanes and EtOAc); HRMS (ESI, $\mathrm{M}^{+}+1$ ) calcd for $\mathrm{C}_{18} \mathrm{H}_{17} \mathrm{O}_{4}$ 297.1127, found 297.1123; ${ }^{1} \mathrm{H}$ NMR (400 MHz, $\mathrm{CDCl}_{3}$ ): $\delta 8.05$ (d, $J 8.8 \mathrm{~Hz}, 2 \mathrm{H}), 7.98$ (br s, $1 \mathrm{H}), 7.97$ (br s, 1H), 7.63 (ddd, $J$ 0.8, 1.6, 8.0 Hz, 1H), 7.50 $(\mathrm{dd}, J 1.6,2.4 \mathrm{~Hz}, 1 \mathrm{H}), 7.41(\mathrm{~d}, J 8.0 \mathrm{~Hz}, 1 \mathrm{H}), 7.17-7.14(\mathrm{~m}, 1 \mathrm{H}), 6.98(\mathrm{~d}, J 8.8 \mathrm{~Hz}, 2 \mathrm{H}), 3.88(\mathrm{~s}$, $3 \mathrm{H}), 3.86(\mathrm{~s}, 3 \mathrm{H}) ;{ }^{13} \mathrm{C}$ NMR $\left(100 \mathrm{MHz}, \mathrm{CDCl}_{3}\right): \delta 189.62,187.86,164.16,159.97,138.24,135.20$, $134.36,131.28(2 \mathrm{x}), 129.92,129.78,121.57,120.50,114.07$ (2x), 112.62, 55.50, 55.43.

1-Biphenyl-4-yl-4-(4-methoxyphenyl)but-2-ene-1,4-dione (3m). $\mathrm{R}_{f} 0.3$ (hexanes : EtOAc 6:1); Yield 91\% (78 mg); Colorless solid; mp 168-169 ${ }^{\circ} \mathrm{C}$ (recrystallized from hexanes and EtOAc); HRMS (ESI, $\mathrm{M}^{+}+1$ ) calcd for $\mathrm{C}_{23} \mathrm{H}_{19} \mathrm{O}_{3} 343.1334$, found 343.1330; ${ }^{1} \mathrm{H} \mathrm{NMR}\left(400 \mathrm{MHz}, \mathrm{CDCl}_{3}\right.$ ): $\delta 8.15(\mathrm{~d}, J 8.8 \mathrm{~Hz}, 2 \mathrm{H}), 8.09(\mathrm{~d}, J 9.2 \mathrm{~Hz}, 2 \mathrm{H}), 8.05(\mathrm{~s}, 2 \mathrm{H}), 7.74(\mathrm{~d}, J 8.8 \mathrm{~Hz}, 2 \mathrm{H}), 7.67-7.63(\mathrm{~m}$, $2 \mathrm{H}), 7.50-7.45(\mathrm{~m}, 2 \mathrm{H}), 7.43-7.39(\mathrm{~m}, 1 \mathrm{H}), 6.99(\mathrm{~d}, J 9.2 \mathrm{~Hz}, 2 \mathrm{H}), 3.90(\mathrm{~s}, 3 \mathrm{H}) ;{ }^{13} \mathrm{C} \mathrm{NMR}(100$ $\left.\mathrm{MHz}_{\mathrm{CDCl}}\right): \delta 189.32,187.90,164.19,146.42,139.57,135.63,135.13,134.35,131.33(2 \mathrm{x})$, 129.98, $129.47(2 x), 128.96(2 x), 128.40,127.43(2 x), 127.27(2 x), 114.11(2 x), 55.54$. 
1-(4-Methoxyphenyl)-4-naphthalen-2-ylbut-2-ene-1,4-dione (3n). $\mathrm{R}_{f} 0.3$ (hexanes : EtOAc 6:1); Yield 87\% (69 mg); Colorless solid; mp 137-138 ${ }^{\circ} \mathrm{C}$ (recrystallized from hexanes and EtOAc); HRMS (ESI, $\mathrm{M}^{+}+1$ ) calcd for $\mathrm{C}_{21} \mathrm{H}_{17} \mathrm{O}_{3} 317.1178$, found $317.1173 ;{ }^{1} \mathrm{H} \mathrm{NMR}\left(400 \mathrm{MHz}, \mathrm{CDCl}_{3}\right.$ ): $\delta 8.60(\mathrm{~d}, J 1.2 \mathrm{~Hz}, 1 \mathrm{H}), 8.19(\mathrm{~d}, J 15.2 \mathrm{~Hz}, 1 \mathrm{H}), 8.14-8.07(\mathrm{~m}, 2 \mathrm{H}), 8.09(\mathrm{~d}, J 8.8 \mathrm{~Hz}, 2 \mathrm{H}), 8.00$ $(\mathrm{d}, J 8.4 \mathrm{~Hz}, 1 \mathrm{H}), 7.94(\mathrm{~d}, J 8.8 \mathrm{~Hz}, 1 \mathrm{H}), 7.90(\mathrm{~d}, J 8.0 \mathrm{~Hz}, 1 \mathrm{H}), 7.65-7.56(\mathrm{~m}, 2 \mathrm{H}), 7.00(\mathrm{~d}, J 8.8$ $\mathrm{Hz}, 2 \mathrm{H}), 3.90(\mathrm{~s}, 3 \mathrm{H}) ;{ }^{13} \mathrm{C} \mathrm{NMR}\left(100 \mathrm{MHz}, \mathrm{CDCl}_{3}\right): \delta 189.61,187.96,164.23,135.85,135.10$, $134.44,134.35,132.46,131.36$ (2x), 131.12, 130.03, 129.75, 128.98, 128.87, 127.83, 127.01, 124.07, 114.13 (2x), 55.55. Single-crystal X-Ray diagram: crystal of compound $3 \mathbf{n}$ was grown by slow diffusion of EtOAc into a solution of compound 3n in $\mathrm{CH}_{2} \mathrm{Cl}_{2}$ to yield colorless prisms. The compound crystallizes in the monoclinic crystal system, space group $\mathrm{P} 21 / \mathrm{n}, a=15.9940(17) \AA, b$ $=5.7255(6) \AA, c=18.2554(18) \AA, V=1567.5(3) \AA^{3}, Z=4, d_{\text {calcd }}=1.340 \mathrm{~g} / \mathrm{cm}^{3}, F(000)=644,2 \theta$ range $1.469 \sim 26.403^{\circ}, \mathrm{R}$ indices (all data) $\mathrm{R} 1=0.1443$, wR2 $=0.1617$.

1-(3-Methoxyphenyl)-4-p-tolylbut-2-ene-1,4-dione (3o). $\mathrm{R}_{f} 0.3$ (hexanes : EtOAc 4:1); Yield 86\% (60 mg); Colorless solid; mp 76-77 ${ }^{\circ} \mathrm{C}$ (recrystallized from hexanes and EtOAc); HRMS (ESI, $\mathrm{M}^{+}+1$ ) calcd for $\mathrm{C}_{18} \mathrm{H}_{17} \mathrm{O}_{3} 281.1178$, found 281.1174; ${ }^{1} \mathrm{H}$ NMR (400 MHz, $\mathrm{CDCl}_{3}$ ): $\delta 7.99$ (br s, 1H), 7.98 (br s, 1H), $7.96(\mathrm{~d}, J 8.0 \mathrm{~Hz}, 2 \mathrm{H}), 7.64(\mathrm{dt}, J 0.8,8.0 \mathrm{~Hz}, 1 \mathrm{H}), 7.56$ (dd, J 1.6, $2.4 \mathrm{~Hz}$, $1 \mathrm{H}), 7.43(\mathrm{~d}, J 8.0 \mathrm{~Hz}, 1 \mathrm{H}), 7.32(\mathrm{~d}, J 8.4 \mathrm{~Hz}, 2 \mathrm{H}), 7.19-7.16(\mathrm{~m}, 1 \mathrm{H}), 3.88(\mathrm{~s}, 3 \mathrm{H}), 2.44(\mathrm{~s}, 3 \mathrm{H})$; ${ }^{13} \mathrm{C}$ NMR (100 MHz, $\left.\mathrm{CDCl}_{3}\right): \delta 189.63,189.25,160.01,144.94,138.23,135.24,134.75,134.38$, 129.82, $129.58(2 \mathrm{x}), 129.01(2 \mathrm{x}), 121.62,120.59,112.64,55.47,21.75$.

1-Biphenyl-4-yl-4-p-tolylbut-2-ene-1,4-dione (3p). $\mathrm{R}_{f} 0.3$ (hexanes : EtOAc 6:1); Yield 93\% (76 $\mathrm{mg}$ ); Colorless solid; mp $154-155{ }^{\circ} \mathrm{C}$ (recrystallized from hexanes and EtOAc); HRMS (ESI, $\mathrm{M}^{+}+1$ ) calcd for $\mathrm{C}_{23} \mathrm{H}_{19} \mathrm{O}_{2} 327.1385$, found 327.1380; ${ }^{1} \mathrm{H} \mathrm{NMR}\left(400 \mathrm{MHz}, \mathrm{CDCl}_{3}\right): \delta 8.15(\mathrm{~d}, J$ $8.8 \mathrm{~Hz}, 2 \mathrm{H}), 8.06(\mathrm{~s}, 2 \mathrm{H}), 7.99$ (d, J $8.4 \mathrm{~Hz}, 2 \mathrm{H}), 7.76$ (d, J 8.4 Hz, 2H), 7.68-7.65 (m, 2H), 7.51$7.40(\mathrm{~m}, 3 \mathrm{H}), 7.34(\mathrm{~d}, J 8.4 \mathrm{~Hz}, 2 \mathrm{H}), 2.45(\mathrm{~s}, 3 \mathrm{H}) ;{ }^{13} \mathrm{C} \mathrm{NMR}\left(100 \mathrm{MHz}, \mathrm{CDCl}_{3}\right): \delta 189.35,189.32$, 146.53, 144.99, 139.62, 135.63, 135.19, 134.75, 134.45, 129.62 (2x), $129.51(2 x), 129.05(2 x)$, $129.00(2 x), 128.44,127.50(2 x), 127.31(2 x), 21.79$.

1-Naphthalen-2-yl-4-p-tolylbut-2-ene-1,4-dione (3q). $\mathrm{R}_{f} 0.3$ (hexanes : EtOAc 6:1); Yield $91 \%$ (68 mg); Colorless solid; mp 126-127 ${ }^{\circ} \mathrm{C}$ (recrystallized from hexanes and EtOAc); HRMS (ESI, $\mathrm{M}^{+}+1$ ) calcd for $\mathrm{C}_{21} \mathrm{H}_{17} \mathrm{O}_{2} 301.1229$, found 301.1225; ${ }^{1} \mathrm{H} \mathrm{NMR}\left(400 \mathrm{MHz}, \mathrm{CDCl}_{3}\right): \delta 8.61(\mathrm{~d}, J$ $0.8 \mathrm{~Hz}, 1 \mathrm{H}), 8.20(\mathrm{~d}, J 15.2 \mathrm{~Hz}, 1 \mathrm{H}), 8.13(\mathrm{dd}, J 2.0,8.8 \mathrm{~Hz}, 1 \mathrm{H}), 8.09$ (d, J $15.2 \mathrm{~Hz}, 1 \mathrm{H}), 8.02-$ $7.99(\mathrm{~m}, 1 \mathrm{H}), 8.01(\mathrm{~d}, J 8.4 \mathrm{~Hz}, 2 \mathrm{H}), 7.95(\mathrm{~d}, J 8.8 \mathrm{~Hz}, 1 \mathrm{H}), 7.90(\mathrm{~d}, J 8.0 \mathrm{~Hz}, 1 \mathrm{H}), 7.66-7.57$ (m, 2H), 7.34 (d, $J 8.4 \mathrm{~Hz}, 2 \mathrm{H}), 2.45$ (s, 3H); ${ }^{13} \mathrm{C} \mathrm{NMR}\left(100 \mathrm{MHz}, \mathrm{CDCl}_{3}\right): \delta 189.59,189.33,145.01$, $135.88,135.12,134.79,134.33,132.47,131.16,131.12,129.77,129.62(2 x), 129.07$ (2x), 129.02, 128.92, 127.86, 127.05, 124.08, 21.79.

1-Biphenyl-4-yl-4-(2,5-dimethoxyphenyl)but-2-ene-1,4-dione (3r). $\mathrm{R}_{f} 0.2$ (hexanes : EtOAc

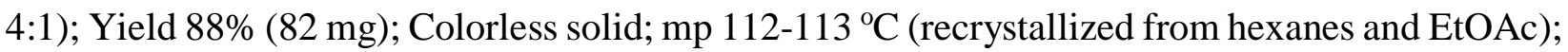
HRMS (ESI, $\mathrm{M}^{+}+1$ ) calcd for $\mathrm{C}_{24} \mathrm{H}_{21} \mathrm{O}_{4} 373.1440$, found $373.1436 ;{ }^{1} \mathrm{H} \mathrm{NMR}\left(400 \mathrm{MHz}, \mathrm{CDCl}_{3}\right.$ ): $\delta 8.13(\mathrm{~d}, J 8.8 \mathrm{~Hz}, 2 \mathrm{H}), 7.93(\mathrm{~d}, J 15.6 \mathrm{~Hz}, 1 \mathrm{H}), 7.87(\mathrm{~d}, J 15.6 \mathrm{~Hz}, 1 \mathrm{H}), 7.74(\mathrm{~d}, J 8.4 \mathrm{~Hz}, 2 \mathrm{H})$, 7.66-7.64 (m, 2H), 7.50-7.46 (m, 2H), 7.44-7.39 (m, 1H), 7.30 (d, J 3.2 Hz, 1H), 7.10 (dd, J 2.8, $8.8 \mathrm{~Hz}, 1 \mathrm{H}), 6.95(\mathrm{~d}, J 8.8 \mathrm{~Hz}, 1 \mathrm{H}), 3.90(\mathrm{~s}, 3 \mathrm{H}), 3.82(\mathrm{~s}, 3 \mathrm{H}) ;{ }^{13} \mathrm{C} \mathrm{NMR}\left(100 \mathrm{MHz}, \mathrm{CDCl}_{3}\right): \delta$ 
191.23, 190.07, 153.69, 153.63, 146.28, 139.74, 139.67, 135.80, 132.68, $129.49(2 x), 128.97(2 x)$, 128.37, 127.95, 127.39 (2x), 127.27 (2x), 121.26, 114.19, 113.29, 56.27, 55.84. Single-crystal XRay diagram: crystal of compound $3 \mathbf{r}$ was grown by slow diffusion of EtOAc into a solution of compound $3 \mathbf{r}$ in $\mathrm{CH}_{2} \mathrm{Cl}_{2}$ to yield colorless prisms. The compound crystallizes in the triclinic crystal system, space group P - 1, $a=7.1811(18) \AA, b=8.2835(19) \AA, c=31.513(8) \AA, V=1829.5(8) \AA^{3}$, $Z=2, d_{\text {calcd }}=1.352 \mathrm{~g} / \mathrm{cm}^{3}, F(000)=784,2 \theta$ range $1.945 \sim 26.551^{\circ}, \mathrm{R}$ indices (all data) $\mathrm{R} 1=0.0578$, $\mathrm{wR} 2=0.1045$.

A representative procedure of compounds $5 \mathrm{a}-\mathrm{e}$ is as follows. LDA (0.5 M in THF, $0.5 \mathrm{~mL}, 0.25$ mmol, commercially available) was added to a solution of $\mathbf{4 a - c}$ and $\mathbf{4 g}(0.25 \mathrm{mmol})$ in anhydrous THF $(5 \mathrm{~mL})$ at $-78{ }^{\circ} \mathrm{C}$. The reaction mixture was stirred at $\mathrm{rt}$ for $5 \mathrm{~min}$. $\alpha$-Bromoacetophenones $\mathbf{1 b}$, 1e and $\mathbf{1 g}(0.25 \mathrm{mmol})$ in THF $(2 \mathrm{~mL})$ were added to the resulting reaction mixture at $-78^{\circ} \mathrm{C}$. The reaction mixture was $-78{ }^{\circ} \mathrm{C}$ for $4 \mathrm{~h}$, warmed to $\mathrm{rt}$, and the solvent was concentrated. The residue was diluted with water $(10 \mathrm{~mL})$ and the mixture was extracted with $\mathrm{CH}_{2} \mathrm{Cl}_{2}$ (3 x $\left.20 \mathrm{~mL}\right)$. The combined organic layers were washed with brine, dried, filtered and evaporated to afford crude product. Purification on silica gel (hexanes/EtOAc $=10 / 1 \sim 3 / 1)$ afforded compounds 5a-e.

1-Phenyl-2-(toluene-4-sulfonyl)-4-p-tolyl-butane-1,4-dione (5a). $\mathrm{R}_{f} 0.2$ (hexanes : EtOAc 4:1); Yield 81\% (82 mg); Colorless solid; mp 170-171 ${ }^{\circ} \mathrm{C}$ (recrystallized from hexanes and EtOAc); HRMS (ESI, $\mathrm{M}^{+}+1$ ) calcd for $\mathrm{C}_{24} \mathrm{H}_{23} \mathrm{O}_{4} \mathrm{~S} 407.1317$, found 407.1311; ${ }^{1} \mathrm{H}$ NMR (400 MHz, $\mathrm{CDCl}_{3}$ ): $\delta$ 7.98-7.95 (m, 2H), 7.81-7.78 (m, 2H), $7.64(\mathrm{~d}, J 8.4 \mathrm{~Hz}, 2 \mathrm{H}), 7.57-7.52(\mathrm{~m}, 1 \mathrm{H}), 7.44-7.40(\mathrm{~m}$, 2H), 7.25-7.23 (m, 4H), 5.69 (dd, J 2.8, $11.2 \mathrm{~Hz}, 1 \mathrm{H}), 4.09$ (dd, $J 11.2,18.0 \mathrm{~Hz}, 1 \mathrm{H}), 3.84$ (dd, $J$ 2.8, $18.0 \mathrm{~Hz}, 1 \mathrm{H}), 2.39$ (s, 3H), 2.38 (s, 3H); $\left.{ }^{13} \mathrm{C} \mathrm{NMR} \mathrm{(100} \mathrm{MHz,} \mathrm{CDCl}_{3}\right): \delta$ 195.03, 191.61, 145.52, 144.86, 136.67, 133.76, 133.50, 132.91, $129.67(2 \mathrm{x}), 129.37(2 \mathrm{x}), 129.30(2 \mathrm{x}), 129.11(2 \mathrm{x})$, $128.46(2 x), 128.28(2 x), 65.74,37.61,21.66,21.59$.

2-(Toluene-4-sulfonyl)-1,4-di-p-tolyl-butane-1,4-dione (5b). $\mathbf{R}_{f} 0.2$ (hexanes : EtOAc 4:1); Yield 75\% (79 mg); Colorless solid; mp 173-174 ${ }^{\circ} \mathrm{C}$ (recrystallized from hexanes and EtOAc); HRMS (ESI, $\mathrm{M}^{+}+1$ ) calcd for $\mathrm{C}_{25} \mathrm{H}_{25} \mathrm{O}_{4} \mathrm{~S} 421.1474$, found 421.1470; ${ }^{1} \mathrm{H} \mathrm{NMR}\left(400 \mathrm{MHz}, \mathrm{CDCl}_{3}\right.$ ): $\delta 7.91$ $(\mathrm{d}, J 8.0 \mathrm{~Hz}, 2 \mathrm{H}), 7.81(\mathrm{~d}, J 8.0 \mathrm{~Hz}, 2 \mathrm{H}), 7.66(\mathrm{~d}, J 8.4 \mathrm{~Hz}, 2 \mathrm{H}), 7.28-7.23(\mathrm{~m}, 6 \mathrm{H}), 5.70(\mathrm{dd}, J 2.8$, $11.2 \mathrm{~Hz}, 1 \mathrm{H}), 4.08$ (dd, J 11.2, $18.0 \mathrm{~Hz}, 1 \mathrm{H}), 3.83$ (dd, J 2.8, $18.0 \mathrm{~Hz}, 1 \mathrm{H}), 2.42$ (s, 3H), 2.40 (s, $6 \mathrm{H}) ;{ }^{13} \mathrm{C} \mathrm{NMR}\left(100 \mathrm{MHz}, \mathrm{CDCl}_{3}\right): \delta 194.95,190.97,145.43,144.74,144.62,134.18,133.70$, $132.92,129.62(2 x), 129.30(2 x), 129.27(4 x), 129.16(2 x), 128.23(2 x), 65.58,37.55,21.63,21.55$ (2x).

4-(2,4-Dimethoxyphenyl)-2-(toluene-4-sulfonyl)-1-p-tolyl-butane-1,4-dione $\quad(\mathbf{5 c}) . \quad \mathrm{R}_{f} \quad 0.3$ (hexanes : EtOAc 2:1); Yield 77\% (90 mg); Colorless solid; mp 172-173 ${ }^{\circ} \mathrm{C}$ (recrystallized from hexanes and EtOAc); HRMS (ESI, $\mathrm{M}^{+}+1$ ) calcd for $\mathrm{C}_{26} \mathrm{H}_{27} \mathrm{O}_{6} \mathrm{~S} 467.1528$, found $467.1523 ;{ }^{1} \mathrm{H}$ NMR (400 MHz, $\left.\mathrm{CDCl}_{3}\right): \delta 7.91(\mathrm{~d}, J 8.0 \mathrm{~Hz}, 2 \mathrm{H}), 7.71(\mathrm{~d}, J 8.8 \mathrm{~Hz}, 1 \mathrm{H}), 7.63(\mathrm{~d}, J 8.0 \mathrm{~Hz}, 2 \mathrm{H})$, $7.24(\mathrm{~d}, J 8.4 \mathrm{~Hz}, 2 \mathrm{H}), 7.21(\mathrm{~d}, J 8.4 \mathrm{~Hz}, 2 \mathrm{H}), 6.42-6.37(\mathrm{~m}, 2 \mathrm{H}), 5.63(\mathrm{dd}, J 2.8,11.2 \mathrm{~Hz}, 1 \mathrm{H})$, $3.91(\mathrm{dd}, J 11.2,18.0 \mathrm{~Hz}, 1 \mathrm{H}), 3.84(\mathrm{~s}, 3 \mathrm{H}), 3.80(\mathrm{dd}, J$ 2.8, $18.0 \mathrm{~Hz}, 1 \mathrm{H}), 3.75(\mathrm{~s}, 3 \mathrm{H}), 2.36$ (s, $3 \mathrm{H}), 2.35$ (s, 3H); ${ }^{13} \mathrm{C}$ NMR (100 MHz, $\left.\mathrm{CDCl}_{3}\right): \delta 193.59,191.28,165.17,161.43,145.16,144.26$, 
134.38, 133.72, 132.78, 129.37 (2x), 129.30 (2x), 129.15 (2x), 129.05 (2x), 118.41, 105.40, 97.89, $65.70,55.37(2 x), 43.32,21.51,21.44$.

4-(2,4-Dimethoxyphenyl)-1-(4-methoxyphenyl)-2-(toluene-4-sulfonyl)butane-1,4-dione (5d). $\mathrm{R}_{f} 0.3$ (hexanes : EtOAc 2:1); Yield 73\% (88 mg); Colorless viscous gum; HRMS (ESI, $\mathrm{M}^{+}+1$ ) calcd for $\mathrm{C}_{26} \mathrm{H}_{27} \mathrm{O}_{7} \mathrm{~S} 483.1478$, found 483.1475; ${ }^{1} \mathrm{H}$ NMR (400 MHz, $\mathrm{CDCl}_{3}$ ): $\delta 8.01(\mathrm{~d}, J 8.8 \mathrm{~Hz}$, 2H), $7.72(\mathrm{~d}, J 8.8 \mathrm{~Hz}, 1 \mathrm{H}), 7.63(\mathrm{~d}, J 8.4 \mathrm{~Hz}, 2 \mathrm{H}), 7.25(\mathrm{~d}, J 8.0 \mathrm{~Hz}, 2 \mathrm{H}), 6.90(\mathrm{~d}, J 8.8 \mathrm{~Hz}, 2 \mathrm{H})$, $6.41(\mathrm{dd}, J 2.4,8.8 \mathrm{~Hz}, 1 \mathrm{H}), 6.38(\mathrm{~d}, J 2.4 \mathrm{~Hz}, 1 \mathrm{H}), 5.62(\mathrm{dd}, J 2.8,11.2 \mathrm{~Hz}, 1 \mathrm{H}), 3.91$ (dd, $J 11.2$, $18.0 \mathrm{~Hz}, 1 \mathrm{H}), 3.86(\mathrm{~s}, 3 \mathrm{H}), 3.82(\mathrm{~s}, 3 \mathrm{H}), 3.81(\mathrm{dd}, J 2.8,18.0 \mathrm{~Hz}, 1 \mathrm{H}), 3.76(\mathrm{~s}, 3 \mathrm{H}), 2.37(\mathrm{~s}, 3 \mathrm{H})$; ${ }^{13} \mathrm{C}$ NMR $\left(100 \mathrm{MHz}, \mathrm{CDCl}_{3}\right): \delta 193.71,189.94,165.18,163.81,161.45,145.15,133.79,132.83$, 131.50 (2x), 129.91, 129.43 (2x), 129.33 (2x), 118.54, 113.64 (2x), 105.40, 97.96, 65.58, 55.41 (3x), 43.23, 21.51 .

1-(2,5-Dimethoxyphenyl)-4-(4-methoxyphenyl)-2-(toluene-4-sulfonyl)-butane-1,4-dione (5e). $\mathrm{R}_{f} 0.3$ (hexanes : EtOAc 2:1); Yield 76\% (92 mg); Colorless solid; mp 147-148 ${ }^{\circ} \mathrm{C}$ (recrystallized from hexanes and EtOAc); HRMS (ESI, $\mathrm{M}^{+}+1$ ) calcd for $\mathrm{C}_{26} \mathrm{H}_{27} \mathrm{O}_{7} \mathrm{~S} 483.1478$, found 483.1480; ${ }^{1} \mathrm{H}$ NMR (400 MHz, $\left.\mathrm{CDCl}_{3}\right): \delta 7.91(\mathrm{~d}, J 8.8 \mathrm{~Hz}, 2 \mathrm{H}), 7.59(\mathrm{~d}, J 8.4 \mathrm{~Hz}, 2 \mathrm{H}), 7.27$ (d, $J 3.2 \mathrm{~Hz}$, $1 \mathrm{H}), 7.16(\mathrm{~d}, J 8.0 \mathrm{~Hz}, 2 \mathrm{H}), 6.98(\mathrm{dd}, J 3.6,9.2 \mathrm{~Hz}, 1 \mathrm{H}), 6.89(\mathrm{~d}, J 8.8 \mathrm{~Hz}, 2 \mathrm{H}), 6.71(\mathrm{~d}, J 8.8 \mathrm{~Hz}$, 1H), 6.42 (dd, J 3.6, $10.4 \mathrm{~Hz}, 1 \mathrm{H}), 4.04$ (dd, $J$ 10.4, $17.2 \mathrm{~Hz}, 1 \mathrm{H}), 3.82$ (s, 3H), 3.77 (s, 3H), 3.75 (s, 3H), 3.74 (dd, J 2.8, $17.2 \mathrm{~Hz}, 1 \mathrm{H}), 2.33$ (s, 3H); ${ }^{13} \mathrm{C}$ NMR (100 MHz, $\mathrm{CDCl}_{3}$ ): $\delta 193.90,190.65$, 163.76, 153.56, 153.27, 144.90, 134.72, 130.41 (2x), 129.06 (2x), $128.94(2 x), 128.79,126.36$, $121.63,114.49,113.70(2 x), 113.46,69.10,56.13,55.65,55.37,36.24,20.91$.

A representative procedure of compounds 7a-d is as follows: 1,2-Diaminobenzenes 6a-d $(0.25$ $\mathrm{mmol})$ were added to a solution of $\mathbf{3 b}(60 \mathrm{mg}, 0.25 \mathrm{mmol})$ in dioxane $(5 \mathrm{~mL})$ at $\mathrm{rt}$. The reaction mixture was stirred at reflux for $4 \mathrm{~h}$, cooled to rt, and the solvent was concentrated. The residue was diluted with water $(10 \mathrm{~mL})$ and the mixture was extracted with $\mathrm{CH}_{2} \mathrm{Cl}_{2}$ (3 x $\left.20 \mathrm{~mL}\right)$. The combined organic layers were washed with brine, dried, filtered and evaporated to afford crude product. Purification on silica gel (hexanes/EtOAc 10/1 3/1) afforded skeleton 7a-d.

2-Phenylquinoxaline (7a). $\mathrm{R}_{f} 0.2$ (hexanes : EtOAc 4:1); Yield 88\% (45 mg); Colorless solid; mp 75-76 ${ }^{\circ} \mathrm{C}$ (recrystallized from hexanes and EtOAc); HRMS (ESI, $\mathrm{M}^{+}+1$ ) calcd for $\mathrm{C}_{14} \mathrm{H}_{11} \mathrm{~N}_{2}$ 207.0922, found 207.0916; ${ }^{1} \mathrm{H}$ NMR (400 MHz, $\left.\mathrm{CDCl}_{3}\right): \delta 9.31(\mathrm{~s}, 1 \mathrm{H}), 8.19-8.10(\mathrm{~m}, 4 \mathrm{H}), 7.78$ $7.70(\mathrm{~m}, 2 \mathrm{H}), 7.57-7.48(\mathrm{~m}, 3 \mathrm{H}) ;{ }^{13} \mathrm{C} \mathrm{NMR}\left(100 \mathrm{MHz}, \mathrm{CDCl}_{3}\right): \delta 151.70,143.21,142.18,141.42$, $136.63,130.19,130.09,129.51,129.45,129.05(2 \mathrm{x}), 128.99,127.45(2 \mathrm{x})$.

6,7-Dimethyl-2-phenylquinoxaline (7b). $\mathrm{R}_{f} 0.2$ (hexanes : EtOAc 4:1); Yield 84\% (49 mg); Colorless solid; mp 130-131 ${ }^{\circ} \mathrm{C}$ (recrystallized from hexanes and EtOAc); HRMS (ESI, $\left.\mathrm{M}^{+}+1\right)$ calcd for $\mathrm{C}_{16} \mathrm{H}_{15} \mathrm{~N}_{2}$ 235.1235, found 235.1232; ${ }^{1} \mathrm{H}$ NMR (400 MHz, $\left.\mathrm{CDCl}_{3}\right): \delta 9.21(\mathrm{~s}, 1 \mathrm{H}), 8.17$ $8.14(\mathrm{~m}, 2 \mathrm{H}), 7.90(\mathrm{~s}, 1 \mathrm{H}), 7.85(\mathrm{~s}, 1 \mathrm{H}), 7.57-7.47$ (m, 3H), 2.50 (br s, 6H); ${ }^{13} \mathrm{C} \mathrm{NMR}(100 \mathrm{MHz}$, $\left.\mathrm{CDCl}_{3}\right): \delta 150.96,142.22,141.19,140.86,140.35,140.19,137.01,129.83,129.04(2 \mathrm{x}), 128.58$, $127.99,127.34(2 \mathrm{x}), 20.36,20.33$.

6,7-Dichloro-2-phenylquinoxaline (7c). $\mathrm{R}_{f} 0.2$ (hexanes : EtOAc 4:1); Yield 86\% (59 mg); Colorless solid; mp $155-156{ }^{\circ} \mathrm{C}$ (recrystallized from hexanes and EtOAc); HRMS (ESI, $\left.\mathrm{M}^{+}+1\right)$ 
calcd for $\mathrm{C}_{14} \mathrm{H}_{9} \mathrm{Cl}_{2} \mathrm{~N}_{2} 275.0143$, found $275.0135 ;{ }^{1} \mathrm{H}$ NMR (400 MHz, $\mathrm{CDCl}_{3}$ ): $\delta 9.32$ (s, $1 \mathrm{H}$ ), 8.28 (s, $1 \mathrm{H}), 8.24(\mathrm{~s}, 1 \mathrm{H}), 8.20-8.17(\mathrm{~m}, 2 \mathrm{H}), 7.61-7.54(\mathrm{~m}, 3 \mathrm{H}) ;{ }^{13} \mathrm{C} \mathrm{NMR}\left(100 \mathrm{MHz}, \mathrm{CDCl}_{3}\right): \delta 152.64$, 144.27, 141.09, 140.24, 135.97, 134.93, 134.01, 130.77, 130.18, 129.77, 129.27 (2x), 127.57 (2x). 2-Phenylbenzo[g]quinoxaline (7d). $\mathrm{R}_{f} 0.2$ (hexanes : EtOAc 4:1); Yield 78\% (50 mg); Colorless solid; mp 158-159 ${ }^{\circ} \mathrm{C}$ (recrystallized from hexanes and EtOAc); HRMS (ESI, $\mathrm{M}^{+}+1$ ) calcd for $\mathrm{C}_{18} \mathrm{H}_{13} \mathrm{~N}_{2} 257.1079$, found 257.1072; ${ }^{1} \mathrm{H}$ NMR $\left(400 \mathrm{MHz}, \mathrm{CDCl}_{3}\right): \delta 9.40(\mathrm{~s}, 1 \mathrm{H}), 8.79(\mathrm{~s}, 1 \mathrm{H})$, $8.75(\mathrm{~s}, 1 \mathrm{H}), 8.29-8.26(\mathrm{~m}, 2 \mathrm{H}), 8.15-8.11(\mathrm{~m}, 2 \mathrm{H}), 7.63-7.56(\mathrm{~m}, 5 \mathrm{H}) ;{ }^{13} \mathrm{C}$ NMR $(100 \mathrm{MHz}$, $\left.\mathrm{CDCl}_{3}\right): \delta 151.68,143.62,138.48,136.89,136.20,134.43,133.84,130.79,129.29(2 \mathrm{x}), 128.56$, $128.52,127.86,127.75(2 x), 127.27,127.14,126.99$.

\section{Acknowledgements}

The authors would like to thank the Ministry of Science and Technology of the Republic of China for its financial support (MOST 105-2113-M-037-001).

\section{Supplementary Material}

${ }^{1} \mathrm{H}$ and ${ }^{13} \mathrm{C}$ NMR $\left(\mathrm{CDCl}_{3}\right)$ spectral data for $\mathbf{3 a - 3 r}, \mathbf{5 a}-5 \mathbf{e}$ and $\mathbf{7 a - 7} \mathbf{d}$ are available as supplementary inormation.

\section{References}

1. El-Awa, A.; Noshi, M. N.; Mollat du Jourdin, X.; Fuchs, P. L. Chem. Rev. 2009, 109, 2315. http://dx.doi.org/10.1021/cr800309r

2. Simpkins, N. S. Sulphones in Organic Synthesis; Pergamon Press: Oxford, UK, 1993.

3. Patai, S.; Rappoport, Z.; Stirling, C., Eds. The Chemistry of Sulphones and Sulphoxides; Wiley: Chichester, UK, 1988.

4. Najera, C.; Yus, M. Tetrahedron 1999, 55, 10547. http://dx.doi.org/10.1016/S0040-4020(99)00600-6

5. Tsui, G. C.; Glenadel, Q.; Lau, C.; Lautens, M. Org. Lett. 2011, 13, 208. http://dx.doi.org/10.1021/o1303291x

6. Chang, M.-Y.; Cheng, Y.-C. Org. Lett. 2015, 17, 5702. http://dx.doi.org/10.1021/acs.orglett.5b01461

7. Xuan, J.; Feng, Z.-J.; Chen, J. R.; Lu, L.-Q.; Xiao, W.-J. Chem. Eur. J. 2014, 20, 3045. http://dx.doi.org/10.1002/chem.201490042 
8. Pandey, G.; Vaitla, J. Org. Lett. 2015, 17, 4890. http://dx.doi.org/10.1021/acs.orglett.5b00097

9. Kuninobu, Y.; Matsuzaki, H.; Nishi, M.; Takai, K. Org. Lett. 2011, 13, 2959. http://dx.doi.org/10.1021/ol2008507

10. Wang, Q.-G.; Zhou, Q.-Q.; Deng, J.-G.; Chen, Y.-C. Org. Lett. 2013, 15, 4786. http://dx.doi.org/10.1021/ol402158u

11. Saraiva, M. T.; Costa, G. P.; Seus, N.; Schumacher, R. F.; Perin, G.; Paixao, M. W.; Luque, R.; Alves, D. Org. Lett. 2015, 17, 6206. http://dx.doi.org/10.1021/acs.orglett.5b03196

12. Kumar, R.; Namboothiri, I. N. N. Org. Lett. 2011, 13, 4016. http://dx.doi.org/10.1021/ol201534f

13. Kumar, R.; Nair, D.; Namboothiri, I. N. N. Tetrahedron 2014, 70, 1794. http:/dx.doi.org/10.1016/j.tet.2014.01.022

14. Bouhlel, A.; Curti, C.; Tabele, C.; Vanelle, P. Molecules 2013, 18, 4293. http://dx.doi.org/10.3390/molecules18044293

15. Chang, M.-Y.; Chen, Y.-C.; Chan, C.-K. Synlett 2014, 25, 1739. http://dx.doi.org/10.1055/s-0033-1339117

16. Chang, M.-Y.; Chen, Y.-C.; Chan, C.-K. Tetrahedron 2014, 70, 8908. http://dx.doi.org/10.1016/j.tet.2014.09.085

17. Chang, M.-Y.; Cheng, Y.-C. Synlett 2016, 27, 854. http://dx.doi.org/10.1055/s-0035-1560423

18. Chang, M.-Y.; Lu, Y.-J.; Cheng, Y.-C. Tetrahedron 2015, 71, 1192. http://dx.doi.org/10.1016/j.tet.2015.01.016

19. Chang, M.-Y.; Chen, Y.-H.; Cheng, Y.-C. Tetrahedron 2016, 72, 518. http://dx.doi.org/10.1016/j.tet.2014.09.085

20. Chang, M.-Y.; Cheng, Y.-C.; Lu, Y.-J. Org. Lett. 2014, 16, 6252. http://dx.doi.org/10.1021/o15032238

21. Chan, C.-K.; Lu, Y.-J.; Chang, M.-Y. Tetrahedron 2015, 71, 9544. http://dx.doi.org/10.1016/j.tet.2015.10.072

22. Chang, M.-Y.; Cheng, Y.-C.; Lu, Y.-J. Org. Lett. 2015, 17, 1264. http://dx.doi.org/10.1021/acs.orglett.5b00246

23. Chang, M.-Y.; Cheng, Y.-C.; Lu, Y.-J. Org. Lett. 2015, 17, 3142. http://dx.doi.org/10.1021/acs.orglett.5b01461

24. Chang, M.-Y.; Lu, Y.-J.; Cheng, Y.-C. Tetrahedron 2015, 71, 6840. http://dx.doi.org/10.1016/j.tet.2015.07.025

25. Chang, M.-Y.; Cheng, Y.-C. Org. Lett. 2016, 18, 608. http://dx.doi.org/10.1021/acs.orglett.5b03696

26. Chang, M.-Y.; Cheng, Y.-C. Org. Lett. 2016, 18, 1682. http://dx.doi.org/10.1021/acs.orglett.6b00603

27. Chang, M.-Y.; Huang, Y.-H.; Wang, H.-S. Tetrahedron 2016, 72, 1888. 
http://dx.doi.org/10.1016/j.tet.2016.02.058

28. Chang, M.-Y.; Chen, Y.-C.; Chan, C.-K. Tetrahedron 2015, 71, 782.

http://dx.doi.org/10.1016/j.tet.2014.12.070

29. Chan, C.-K.; Chen, Y.-C.; Chen, Y.-L.; Chang, M.-Y. Tetrahedron 2015, 71, 9187.

http://dx.doi.org/10.1016/j.tet.2015.10.060

30. Salva, J.; Faulkner, D. J. J. Org. Chem. 1990, 55, 1941.

http://dx.doi.org/10.1021/jo00293a047

31. Ballini, R.; Astolfi, P. Liebigs Ann. 1996, 1879.

http://dx.doi.org/10.1002/jlac.199619961124

32. Meng, X.; Zhang, J.; Chen, G.; Chen, B.; Zhao, P. Catal. Commun. 2015, 69, 239.

http://dx.doi.org/10.1016/j.catcom.2015.07.003

33. Li, S.-Y.; Wang, X.-B.; Jiang, N.; Kong, L.-Y. Eur. J. Org. Chem. 2014, 8035.

http://dx.doi.org/10.1002/ejoc.201403236

34. Xu, K.; Fang, Y.; Yan, Z.; Zha, Z.; Wang, Z. Org. Lett. 2013, 15, 2148.

http://dx.doi.org/10.1021/o14006344

35. Gao, M.; Yang, Y.; Wu, Y.-D.; Deng, C.; Cao, L.-P.; Meng, X.-G.; Wu, Z.-X. Org. Lett. 2010 , $12,1856$.

http://dx.doi.org/10.1021/ol100473f

36. Yin, G.; Zhou, B.; Meng, X.; Wu, Z.-X.; Pan, Y. Org. Lett. 2006, 8, 2245.

http://dx.doi.org/10.1021/ol060541e

37. Crone, B.; Kirsch, S. F. Chem. Commun. 2006, 764.

http://dx.doi.org/10.1039/B515838A

38. Yu, J.-Q.; Corey, E. J. J. Am. Chem. Soc. 2003, 125, 3232.

http://dx.doi.org/10.1021/ja0340735

39. Runcie, K. A.; Taylor, R. J. K. Chem. Commun. $2002,974$. http://dx.doi.org/10.1039/B201513G

40. CCDC 1469126 (3n) and 1469127 (3r) contain the supplementary crystallographic data for this paper. This data can be obtained free of charge via www.ccdc.cam.ac.uk/conts/retrieving.html (or from the CCDC, 12 Union Road, Cambridge CB2 1EZ, UK; fax: 44-1223-336033; e-mail: deposit@ccdc.cam.ac.uk).

41. Song, H. J.; Lee, T. H.; Han, M. H.; Lee, J. Y.; Moon, D. K. Polymer 2013, 54, 1072. http://dx.doi.org/10.1016/j.polymer.2012.10.056

42. Shi, J.; Chai, Z.; Su, J.; Chen, J.; Tang, R.; Fan, K.; Zhang, L.; Han, H.; Qin, J.; Peng, T.; Li, Q.; Li, Z. Dyes Pigments 2013, 98, 405.

http://dx.doi.org/10.1016/j.dyepig.2013.03.015 
43. Rodrigues, F. A. R.; Bomfim, I. da S.; Cavalcanti, B. C.; Pessoa, C. do O.; Wardell, J. L.; Wardell, S. M. S. V.; Pinheiro, A. C.; Kaiser, C. R.; Nogueira, T. C. M.; Low, J. N.; Gomes, L. R.; de Souza, M. V. N. Bioorg. Med. Chem. Lett. 2014, 24, 934. http://dx.doi.org/10.1016/j.bmcl.2013.12.074

44. Hui, X.; Desrivot, J.; Bories, C.; Loiseau, P. M.; Franck, X.; Hocquemiller, R.; Figadere, B. Bioorg. Med. Chem. Lett. 2006, 16, 815. http://dx.doi.org/10.1016/j.bmcl.2005.11.025

45. Chang, M. Y.; Lee, T. W.; Hsu, R. T.; Yen, T. L. Synthesis 2011, 19, 3143 and references cited therein. http://dx.doi.org/10.1055/s-0030-1260147

46. Yang, Y.; Ni, F.; Shu, W.-M.; Wu, A.-X. Chem. Eur. J. 2014, 20, 11776. http://dx.doi.org/10.1002/chem.201403001 